\section{SCIENCE CHINA \\ Physics, Mechanics \& Astronomy}

- Research Highlight .

August 2021 Vol. 64 No. 8: 281031

https://doi.org/10.1007/s11433-021-1679-4

\title{
Improved constraints on dark matter effective interactions from CDEX
}

\author{
Yu-Feng Zhou ${ }^{1,2 *}$ \\ ${ }^{1}$ CAS Key Laboratory of Theoretical Physics, Institute of Theoretical Physics, Chinese Academy of Sciences, Beijing 100190, China; \\ ${ }^{2}$ University of Chinese Academy of Sciences, Beijing 100190, China
}

Received February 8, 2021; accepted February 9, 2021; published online May 6, 2021

Citation: Y.-F. Zhou, Improved constraints on dark matter effective interactions from CDEX, Sci. China-Phys. Mech. Astron. 64, 281031 (2021),

https://doi.org/10.1007/s11433-021-1679-4

Numerous astrophysical observations have established a paradigm that the dominant component of matter in the Universe should be non-luminous and non-baryonic, which is often referred to as dark matter (DM) [1]. Up to now, the particle nature of DM, such as its mass and interactions, remains largely unknown. Studies on popular DM candidates suggest that DM may interact with ordinary matter with a strength reachable by modern experimental technologies. This has thus triggered enormous experimental efforts to search for DM non-gravitational interactions. A kind of DM search experiment called DM direct search experiment is focused on detecting tiny recoil energy from possible direct scatterings between DM and a target nucleus [2]. There are more than thirty on-going such experiments around the world, including two in China, i.e., the CDEX and PandaX experiments.

The interpretation of the current DM direct detection data is usually model-dependent, which complicates the corresponding analyses, because even for the same experimental data, the constraints obtained for one model cannot be applied directly to another one. A possible approach for improvement is to set constraints in a less model-dependent way by effective theories, each of which contains all possible interactions allowed by given symmetric principles with interaction strength (Wilson coefficients) left as free parameters to be constrained solely by the data.
In a recent study carried out by the CDEX Collaboration [3], two effective theories were adopted for data analyses. One is the non-relativistic effective theory for the DMnucleus scattering process based on the Galilean relativity [4], and the other is the chiral effective field theory based on chiral symmetry [5]. The reseach team made use of two data sets from CDEX-1B and CDEX-10, and performed both the time-integrated and time-dependent data analysis

Via these analyses, they derived new limits on the cross sections for DM-nucleon scatterings towards lower DM masses. These limits are among the most stringent limits derived from DM direct detection experiments.

1 G. Bertone, and D. Hooper, Rev. Mod. Phys. 90, 045002 (2018), arXiv: 1605.04909; J. L. Feng, Annu. Rev. Astron. Astrophys. 48, 495 (2010), arXiv: 1003.0904.

2 M. Schumann, J. Phys. G-Nucl. Part. Phys. 46, 103003 (2019), arXiv: 1903.03026

3 Y. Wang, et al. (CDEX Collaboration), Sci. China-Phys. Mech. Astron. 64, 281011 (2021), arXiv: 2007.15555.

4 J. J. Fan, M. Reece, and L. T. Wang, J. Cosmol. Astropart. Phys. 2010(11), 042 (2010), arXiv: 1008.1591; A. L. Fitzpatrick, W. Haxton, E. Katz, N. Lubbers, and Y. Xu, J. Cosmol. Astropart. Phys. 2013(02), 004 (2013), arXiv: 1203.3542.

5 E. Epelbaum, H. W. Hammer, and U. G. Meißner, Rev. Mod. Phys. 81, 1773 (2009), arXiv: 0811.1338.

*Corresponding author (email: yfzhou@itp.ac.cn) 